\title{
Complications of mid urethral slings: important outcomes for future clinical trials
}

Daneshgari F, Kong W, Swartz M

Department of Urology and Female Pelvic Surgery, Upstate Medical University, Syracuse, New York, USA

J Urol. 2008; 180: 1890-7

Purpose: Mid urethral slings are becoming the first line surgical treatment for stress urinary incontinence in women. We reviewed the complications of mid urethral sling placement and their potential pathophysiology.

Materials and Methods: We conducted a literature search on MEDLINE from 1995 to 2007 using the key words sling, complications, mid-urethral slings, transvaginal tape, transobturator tape, trials, pathophysiology and complications. The Cochrane database was also searched. The results were summarized according to the type of mid urethral slings reported.

Results: There were 928 MEDLINE citations for sling and complications, 279 for sling and complications and bladder, and 68 for sling and complications and voiding dysfunction. The reported complication rates ranged from $4.3 \%$ to $75.1 \%$ for retropubic and $10.5 \%$ to $31.3 \%$ for transobturator mid urethral slings. Complications included bladder perforation, hemorrhage, bowel injury, vaginal extrusion, de novo urgency and urge incontinence, urinary tract infections and voiding dysfunction. Retropubic mid urethral slings led to a higher occurrence of complications such as bladder perforation and hematoma. In addition, the retropubic approach resulted in serious complications such as bowel injury, major vascular injury and death. Groin pain was more common after the transobturator approach. Experimental studies indicated that the potential mechanisms for sling complications may include vaginal dissection, denervation injury and bladder remodeling.

Conclusions: Mid urethral slings result in bothersome complications which should not be minimized. Awareness of these complications should encourage improvements in patient counseling as well as further investigation of the underlying mechanisms. Decreasing complications should be considered an important outcome for future clinical studies of mid urethral slings.

\section{Editorial Comment}

A noted authority in the field presents a broad spanning review of potential complications of what has probably become the most popular method of treating female stress urinary incontinence. Though the authors did not report any instances of bowel perforation with a transobturator sling, our institution is preparing a case report on a colon injury associated with a transobturator tape procedure so this complication may and does occur. If history serves as a guide, with the increased used of mid urethral slings the breath and depth of complications reported will expand. Of note is the somewhat high rate of new onset urinary urge incontinence associated with a retropubic mid urethral sling. This relatively high rate of urinary urgency was previously noted in the past when reviewing the work of Alperin et al (1). This rate of de novo urgency should be taken in historical context and compared to discussions on this occurrence in the past (2).

\section{References}

1. Alperin M, Abrahams-Gessel S, Wakamatsu MM: Development of de novo urge incontinence in women post sling: the role of preoperative urodynamics in assessing the risk. Neurourol Urodyn. 2008; 27: 407-11.

2. McGuire E: Bladder instability and stress incontinence. Neurourol Urodyn. 1988; 7: 563-7.

\author{
Dr. Steven P. Petrou \\ Professor of Urology, Associate Dean \\ Mayo School of Graduate Medical Education \\ Jacksonville, Florida, USA \\ E-mail:petrou.steven@mayo.edu
}

